\title{
Multiflows in Multi-Channel Multi-Radio Multihop Wireless Networks
}

\author{
Peng-Jun Wan*, Yu Cheng ${ }^{\dagger}$, Zhu Wang* and Frances Yao ${ }^{\ddagger}$ \\ * Department of Computer Science, Illinois Institute of Technology \\ Emails:wan@cs.iit.edu, zwang59@iit.edu \\ $\dagger$ Department of Electrical and Computer Engineering, Illinois Institute of Technology \\ Email: cheng@iit.edu \\ $\ddagger$ Department of Computer Science, City University of Hong Kong \\ Email: csfyao@cityu.edu.hk
}

\begin{abstract}
This paper studies maximum multiflow (MMF) and maximum concurrent multiflow (MCMF) in muliti-channel multi-radio multihop wireless networks under the 802.11 interference model or the protocol interference model. We introduce a fine-grained network representation of multi-channel multi-radio multihop wireless networks and present some essential topological properties of its associated conflict graph. By exploiting these properties, we develop practical polynomial approximation algorithms for MMF and MCMF with constant approximation bounds regardless of the number of channels and radios. Under the 802.11 interference model, their approximation bounds are at most 20 in general and at most 8 with uniform interference radii; under the protocol interference model, if the interference radius of each node is at least $c$ times its communication radius, their approximation bounds are at most $2\left(\left[\pi / \arcsin \frac{c-1}{2 c}\right]+1\right)$. In addition, we also prove that if the number of channels is bounded by a constant (which is typical in practical networks), both MMF and MCMF admit a polynomial-time approximation scheme under the $\mathbf{8 0 2 . 1 1}$ interference model or under the protocol interference model with some additional mild conditions.
\end{abstract}

Index Terms-Link scheduling, multi-channel multi-radio, maximum (concurrent) multiflow, approximation algorithm.

\section{INTRODUCTION}

With the rapid technology advances, many off-the-shelf wireless transceivers (i.e., radios) are capable of operating on multiple channels. For example, the IEEE $802.11 \mathrm{~b} / \mathrm{g}$ standard and IEEE 802.11a standard provide 3 and 12 channels respectively, and MICA2 sensor motes support more than 50 channels. The rapidly diminishing prices of the radios has also made it feasible to equip a wireless node with multiple radios. Providing each node with one or more multichannel radios offers a promising avenue for enhancing the network capacity by simultaneously exploiting multiple nonoverlapping channels through different radio interfaces and mitigating interferences through proper channel assignment. However, it is quite challenging to effectively utilize multiple channels and/or multiple radios to maximize throughput capacity. Throughput maximization in multi-channel multiradio (MC-MR) multihop wireless networks involves four correlated subproblems in the top-down order. First, for each end-to-end communication request, we need to find a group of routing paths and compute the amount of traffic carried over each of these paths. Second, for each communication link, we have to properly distribute its total traffic demand to all possible radio-links joining the two end nodes of this communication links. Third, for each radio-link, we need to further split its traffic demand to be transmitted over individual channels. Forth, for each radio-link over each channel, we must assign a sequence of transmitting time-intervals subject to the constraint that at any time all scheduled links can transmit successfully. Therefore, a joint solution should be developed for routing, radio assignment, channel assignment, and transmission scheduling altogether.

In this paper, we present approximation algorithms for computing a maximum (concurrent) multiflow in MC-MR multihop wireless networks under either the 802.11 interference model or protocol interference model. An instance of such network is specified by a finite planar set $V$ of nodes together with a communication radius function $r \in \mathbb{R}_{+}^{V}$, an interference radius function $\rho \in \mathbb{R}_{+}^{V}$ satisfying that $\rho \geq r$, a radio configuration function $\tau \in \mathbb{N}^{V}$, and the number of available channels $\lambda$ satisfying that $\lambda \geq \max _{v \in V} \tau(v)$. The communication (respectively, interference) range of each radio at a node $v \in V$ is the disk centered at $v$ of radius $r(v)$ and $\rho(v)$ respectively. Under the 802.11 interference model, the transmission from the $i$-th radio at a node $u$ to the $j$-th radio at a node $v$ over the $k$-th channel succeeds if

1) $u$ and $v$ are within each other's communication range,

2) neither the $i$-th radio at $u$ and the $j$-th radio at $v$ is involved in any transmission or reception,

3) neither of $u$ and $v$ is within the interference range of any other node with a radio transmitting or receiving at the same time over the $k$-th channel.

Under the protocol interference model, the transmission from the $i$-th radio at a node $u$ to the $j$-th radio at a node $v$ over the $k$-th channel succeeds if

1) $v$ is within the communication range of $u$,

2) neither the $i$-th radio at $u$ and the $j$-th radio at $v$ is involved in any transmission or reception,

3) $v$ is not within the interference range of any other node with a radio transmitting at the same time over the $k$-th channel. 
Since it is NP-hard to compute a maximum (concurrent) multiflow in single-channel, single-radio (SC-SR) multihop wireless networks even when all nodes have uniform (and fixed) communication radii and uniform (and fixed) interference radii [13], it is also NP-hard to compute a maximum (concurrent) multiflow in the more general MC-MR multihop wireless networks even when all nodes have uniform (and fixed) communication radii and uniform (and fixed) interference radii. In this paper, we introduce a fine-grained network representation of MC-MR multihop wireless networks and develop some useful topological properties of the conflict graph associated with this network representation. By exploiting these properties, we devise practical polynomial approximation algorithms whose approximation bounds are constants regardless of the number of channels and radios. Specifically, under the 802.11 interference model, we develop 20-approximation algorithm with arbitrary interference radii, and 8-approximation algorithms with uniform interference radii. Under the protocol interference model, we develop $2\left(\left\lceil\pi / \arcsin \frac{c-1}{2 c}\right\rceil+1\right)$-approximation algorithms where $c=$ $\min _{v \in V} \rho(v) / c(v)$. In addition, we also show that if the number of channels $\lambda$ is bounded by a constant (which is typical in practical networks), there exists a PTAS under the 802.11 interference model or under the protocol interference model with some additional mild conditions. In other words, for any fixed $\varepsilon>0$, there is a polynomial-time (depending on $\varepsilon)(1+\varepsilon)$-approximation algorithm for maximum (concurrent) multiflow.

The remainder of this paper is organized as follows. In Section II, we introduce two polynomial approximate independence polytopes of an arbitrary graph. In Section III, we describe a fine-grained network representation of MC-MR multihop wireless networks and prove some essential topological properties of its associated conflict graph. In Section IV, we present our polynomial approximation algorithms. In Section $\mathrm{V}$, we review the literature on maximum (concurrent) multiflow in multihop wireless networks under either the 802.11/protocol interference model. In Section VI, we summarize the paper and describe two generalizations.

At the end of this section, we introduce some standard notations and terms used throughout this paper. Let $S$ be a finite subset. For any real function $f \in \mathbb{R}^{S}$ and any subset $S^{\prime} \subseteq S, f\left(S^{\prime}\right)$ denotes $\sum_{e \in S^{\prime}} f(e)$. Let $G$ be an undirected graph with vertex set $V$. For any subset $U$ of $V, G[U]$ denotes the subgraph of $G$ induced by $U$. For any node $v \in V, N_{G}(v)$ denotes the set of neighbors of $v$ in $G$, and $N_{G}[v]$ denotes $\{v\} \cup N_{G}(v)$. Consider a vertex ordering $\prec$ of $V$. For each $v \in V, N_{G}^{\prec}(v)$ denotes the set of neighbors of $v$ preceding $v$ in the ordering $\prec$, and $N_{G}^{\prec}[v]$ denotes $\{v\} \cup N_{G}^{\prec}(v)$. Suppose that $D$ is digraph with vertex set $V$. For any subset $U$ of $V$, $D[U]$ denotes the subgraph of $D$ induced by $U$. For each $v \in V, N_{D}^{i n}(v)$ denotes the set of in-neighbors of $v$ in $D$, and $N_{D}^{i n}[v]$ denotes $\{v\} \cup N_{D}^{i n}(v)$.

\section{INDEPENDENCE POLYTOPES}

Let $G=(V, E)$ be an undirected graph. A subset $I$ of $V$ is an independent set (IS) of $G$ if no two nodes in $I$ are adjacent. If $I$ is a independent set of $G$ but no proper superset of $I$ is a independent set of $G$, then $I$ is called a maximal independent set of $G$. Any node ordering $\left\langle v_{1}, v_{2}, \cdots, v_{n}\right\rangle$ of $V$ induces a maximal IS $I$ in the following first-fit manner: Initially, $I=\left\{v_{1}\right\}$. For $i=2$ up to $n$, add $v_{i}$ to $I$ if $v_{i}$ is not adjacent to any node in $I$. An independent set of the largest size is called a maximum independent set. Let $\mathcal{I}$ be the collection of all independent sets of $G$. For any vertex ordering $\prec$ of $G$, its backward local independence number (BLIN) in $G$ is defined to be the parameter

$$
\max _{v \in V} \max \left\{|I|: I \subseteq N_{G}^{\prec}[v], I \in \mathcal{I}\right\} .
$$

An orientation of $G$ is a digraph obtained from $G$ by imposing an orientation on each edge of $G$. For any orientation $D$ of $G$, its inward local independence number (ILIN) in $G$ is defined to be the parameter

$$
\max _{v \in V} \max \left\{|I|: I \subseteq N_{D}^{i n}[v], I \in \mathcal{I}\right\} .
$$

For any $d \in \mathbb{R}_{+}^{V}$, a fractional (weighted) coloring of $(G, d)$ is a set of $k$ pairs $\left(I_{j}, \ell_{j}\right) \in \mathcal{I} \times \mathbb{R}_{+}$for $1 \leq j \leq k$ satisfying that for each $v \in V$,

$$
\sum_{j=1}^{k} \ell_{j}\left|I_{j} \cap\{v\}\right|=d(v) .
$$

The two values $k$ and $\sum_{j=1}^{k} \ell_{j}$ are referred to as the number and length of the coloring respectively. The fractional chromatic number $\chi_{f}(G, d)$ of $(G, d)$ is defined as the minimum length of all fractional colorings of $(G, d)$. A first-fit fractional weighted coloring algorithm was developed in [13], which is described as follows. Consider a vertex ordering $\prec$ of $G$. For any $d \in \mathbb{R}_{+}^{V}$, this algorithm produces a fractional coloring $S$ of $(G, d)$ in the following iterative manner. Initially, $S$ is empty. In each iteration, let $V^{\prime}$ be the subset of vertices $v \in V$ with $d(v)>0$. Compute a maximal set $I$ of independent vertices in $V^{\prime}$ in the first-fit manner in the ordering $\prec$ restricted to $V^{\prime}$. Let $\ell=\min _{v \in I} d(v)$, and add $(I, \ell)$ to $S$. For each $v \in I$, replace $d(v)$ by $d(v)-\ell$. Repeat this iteration until $d=0$. The output $S$ is referred to as the first-fit fractional coloring of $(G, d)$ in the ordering $\prec$. Since in each iteration the number of vertices $v$ with $d(v)>0$ strictly decreases, the number of iterations, or the number of colors, is bounded by $n$. In addition, if $d$ is integral then the output $S$ is also integral. In addition, it was proved in [13] that the length of $\Pi$ is at most $\max _{v \in V} d\left(N_{G}^{\prec}[v]\right)$.

The independence polytope $P$ of $G$ is the convex hull of the incidence vectors of the independent sets in $\mathcal{I}$. Equivalently, it consists of all $d \in \mathbb{R}_{+}^{V}$ with $\chi_{f}(G, d) \leq 1$. A subset $Q$ of $\mathbb{R}_{+}^{V}$ is called a polynomial $\mu$-approximate independence polytope 
of $G$ for some $\mu \geq 1$ if it satisfies the three conditions: (1) $Q$ has an explicit representation by a polynomial number of linear inequalities or equalities, (2) for any $d \in Q$, a fractional coloring of of $(G, d)$ with length at most one can be computed in polynomial time, and (3) $P \subseteq \mu Q$. Note that the second condition implies that $Q \subseteq P$. Two polynomial approximate independence polytopes of $G$ were introduced in [13]. For any vertex ordering $\prec$ of $G$, its backard independence polytope of $G$ is defined to be

$$
\left\{d \in \mathbb{R}_{+}^{V}: \max _{v \in V} d\left(N_{G}^{\prec}[v]\right) \leq 1\right\} .
$$

For any orientation $D$ of $G$, its inward independence polytope of $G$ is defined to be

$$
\left\{d \in \mathbb{R}_{+}^{V}: \max _{v \in V}\left(d(v)+2 d\left(N_{D}^{i n}(v)\right)\right) \leq 1\right\} .
$$

The following theorem was proved in [13].

Theorem 1: Consider a graph $G=(V, E)$.

1) For any vertex ordering of $G$ with BLIN $\mu$, its backward independence polytope of $G$ is a polynomial $\mu$ approximate independence polytope of $G$.

2) For any orientation of $G$ with ILIN $\mu$, its inward independence polytope of $G$ is a polynomial $2 \mu$-approximate independence polytope of $G$.

\section{A Fine-Grained Network REPRESENTATION}

An instance of MC-MR multihop wireless network is specified by a finite planar set $V$ of nodes together with a communication radius function $r \in \mathbb{R}_{+}^{V}$, an interference radius function $\rho \in \mathbb{R}_{+}^{V}$ satisfying that $\rho \geq r$, a radio configuration function $\tau \in \mathbb{N}^{V}$, and the number of available channels $\lambda$ satisfying that $\lambda \geq \max _{v \in V} \tau(v)$. The communication (respectively, interference) range of each radio at a node $v \in V$ is the disk centered at $v$ of radius $r(v)$ and $\rho(v)$ respectively. Each communication link in $A$ is encoded by an ordered quintuple of the transmitting node, the receiving node, the radio at the transmitting node, the radio at the receiving node, and the channel. The set $A$ of communication links is better defined with the help of the set $A^{*}$ of communication links at the node level, which is defined as follows:

- 802.11 interference model: $A^{*}$ consists of all pairs $(u, v)$ satisfying that $u$ and $v$ are within each other's communications ranges. Two links in $A^{*}$ conflict with each other if and only if at least one link has an end lying in the interference range of some endpoint of the other link.

- Protocol interference model: $A^{*}$ consists of all pairs $(u, v)$ satisfying that $v$ is within the communication range of $u$. Two links in $A^{*}$ conflict with each other if and only if the receiving end of at least one link lies in the interference range of the transmitting end of the other link.
Under either of the two interference models, the node-level communication topology of $V$ is given by the digraph $\left(V, A^{*}\right)$, and the conflict graph $G^{*}$ of $A^{*}$ is the undirected graph on $A^{*}$ in which two links are adjacent if and only if they conflict with each other. We denote by $\mathcal{I}^{*}$ the collection of the independent sets in the conflict graph $G^{*}$. Note that the triple $\left(V, A^{*}, \mathcal{I}^{*}\right)$ essentially represents the SC-SR multihop wireless network.

For each link $e=(u, v) \in A^{*}$, we make $\lambda \cdot \tau(u) \cdot \tau(v)$ replications $\left(u, v, \gamma_{1}, \gamma_{2}, \gamma_{3}\right)$ for $1 \leq \gamma_{1} \leq \tau(u), 1 \leq \gamma_{2} \leq$ $\tau(v)$, and $1 \leq \gamma_{2} \leq \lambda$. A replication $\left(u, v, \gamma_{1}, \gamma_{2}, \gamma_{3}\right)$ of $e$ always utilizes the $\gamma_{1}$-th radio at $u$ and the $\gamma_{2}$-th radio at $v$ over the $\gamma_{3}$-th channel. Each of these replications is referred to as a radio-level link of $e$, and conversely $e$ is referred to as the node-level link of all its replications. Then, the set $A$ of radio-level links consists of all replications of the links in $A^{*}$. The radio-level communication topology of $V$ is given by the directed multi-graph $(V, A)$ obtained from $\left(V, A^{*}\right)$ by replace each link $e \in A^{*}$ with its replications. Two links in $A$ conflict with each other if and only if either they share a common radio or otherwise they share a common channel and their node-level links either are the same or conflict with each other. The conflict graph $G$ of $A$ is the undirected graph on $A$ in which two links are adjacent if and only if they conflict with each other. We denote by $\mathcal{I}$ the collection of the independent sets in the conflict graph $G$. The triple $(V, A, \mathcal{I})$ is referred to as the fine-grained network representation.

We distinguish two types of conflicts among the links in $A$. A pair of conflicting links in $A$ are said to have primary conflict if there share at least one common radio, and are said to have secondary conflict otherwise. Clearly, any pair of links with secondary conflict must share a common channel. In addition, their node-level links either are the same or conflict with each other. For any pair of conflicting links $a$ and $a^{\prime}$ in $A$ with distinct node-level links $e$ and $e^{\prime}$, the two links $e$ and $e^{\prime}$ also conflict with each other. Indeed, if $a$ and $a^{\prime}$ have the primary conflict, then $e$ and $e^{\prime}$ share a common endpoint and hence conflict with each other; if $a$ and $a^{\prime}$ have the secondary conflict, then $e$ and $e^{\prime}$ also conflict with each. For any pair of conflict-free links in $A$ with a common channel, their nodelevel links must be distinct and conflict-free. In general, for any independent set of links in $A$ sharing a common channel, their node-level links are distinct and form an independent set in $\mathcal{I}^{*}$.

We present some intrinsic relations between $G$ and $G^{*}$. Consider an ordering $\prec$ of $A$ and an ordering $\lessdot$ of $A^{*}$. They are said to be compatible if for any pair of conflicting links $a$ and $a^{\prime}$ in $A$ with distinct node-level links $e$ and $e^{\prime}$ respectively, $a \prec a^{\prime}$ if and only if $e \lessdot e^{\prime}$. For any ordering $\lessdot$ of $A^{*}$, a compatible ordering of $A$ can be constructed follows: (1) For any pair of links $e$ and $e^{\prime}$ in $A^{*}$ with $e \lessdot e^{\prime}$, all replications of $e$ are sorted before all replications of $e^{\prime}$; (2) for each $e \in A^{*}$, all replications of $e$ are sorted arbitrarily. 
Lemma 2: Suppose that $\lessdot$ is an ordering of $A^{*}$ and its BLIN is $\mu$. Then for any ordering $\prec$ of $A$ compatible with $\lessdot$, its BLIN is at most $\mu+2$.

Proof: Consider any link $a \in A$. Let $I$ be any independent set of its preceding neighbors. We partition $I$ into two subsets $I_{1}$ and $I_{2}$ as follows. $I_{1}$ consists of all links in $I$ having primary conflict with $a$, and $I_{2}$ consists of all links in $I$ having secondary conflict with $a$. Since all links in $I_{1}$ either share the transmitting radio with $a$ or share the receiving radio with $a$, $\left|I_{1}\right| \leq 2$. Now, we show that $\left|I_{2}\right| \leq \mu$. If $\left|I_{2}\right| \leq 1$, then $\left|I_{2}\right| \leq \mu$ trivially. So we assume that $\left|I_{2}\right| \geq 2$. Since all links in $I_{2}$ have the secondary conflict with $a$, they all share the same channel as $a$. Hence, their node-level links are all distinct and form an independent set in $\mathcal{I}^{*}$. Let $I_{2}^{*}$ denote the set of their node-level links. Then, $\left|I_{2}^{*}\right|=\left|I_{2}\right| \geq 2$. Let $e$ be the node-level link of $a$. Then, every link in $I_{2}^{*} \backslash\{e\}$ conflicts with $e$. Therefore, $e \notin I_{2}^{*}$. By the compatibility of the ordering $\prec$ with $\lessdot$, all links in $I_{2}^{*}$ precede $e$ in the ordering $\lessdot$. Thus, $\left|I_{2}^{*}\right| \leq \mu$ and hence $\left|I_{2}\right| \leq \mu$. Therefore,

$$
|I|=\left|I_{1}\right|+\left|I_{2}\right| \leq \mu+2 \text {. }
$$

So, the lemma follows.

Consider an orientation $D$ of $G$ and an orientation $D^{*}$ of $G^{*}$. They are said to be compatible if for any pair of conflicting links $a$ and $a^{\prime}$ in $A$ with distinct node-level links $e$ and $e^{\prime}$ respectively, $a$ is an in-neighbor of $a^{\prime}$ in $D$ if and only $e$ is an in-neighbor of $e^{\prime}$ in $D^{*}$. For any orientation $D^{*}$ of $G^{*}$, a compatible orientation $D$ of $G$ can be constructed follows. Consider any conflicting pair of links $a$ and $a^{\prime}$ in $A$. Let $e$ and $e^{\prime}$ be their respective node-level links. If $e \neq e^{\prime}$, we take the orientation from $a$ to $a^{\prime}$ in $D$ in $D$ if and only if $\left(e, e^{\prime}\right)$ is an arc in $D^{*}$. If $e=e^{\prime}$, then we take an arbitrary orientation of the edge between $a$ to $a^{\prime}$ in $D$.

Lemma 3: Suppose that $D^{*}$ is an orientation of $G^{*}$ and its ILIN is $\mu$. Then for any orientation $D$ of $G$ compatible with $D^{*}$, its ILIN is at most $\mu+2$.

Proof: Consider any link $a \in A$. Let $I$ be any independent set of in-neighbors in $G$. We partition $I$ into two subsets $I_{1}$ and $I_{2}$ as follows. $I_{1}$ consists of all links in $I$ having primary conflict with $a$, and $I_{2}$ consists of all links in $I$ having secondary conflict with $a$. Since all links in $I_{1}$ either share the transmitting radio with $a$ or share the receiving radio with $a$, $\left|I_{1}\right| \leq 2$. Now, we show that $\left|I_{2}\right| \leq \mu$. If $\left|I_{2}\right| \leq 1$, then $\left|I_{2}\right| \leq \mu$ trivially. So we assume that $\left|I_{2}\right| \geq 2$. Since all links in $I_{2}$ have the secondary conflict with $a$, they all share the same channel as $a$. Hence, their node-level links are all distinct and form an independent set in $\mathcal{I}^{*}$. Let $I_{2}^{*}$ denote the set of their node-level links. Then, $\left|I_{2}^{*}\right|=\left|I_{2}\right| \geq 2$. Let $e$ be the node-level link of $a$. Then, every link in $I_{2}^{*} \backslash\{e\}$ conflicts with $e$. Therefore, $e \notin I_{2}^{*}$. By the compatibility of $D$ and $D^{*}$, all links in $I_{2}^{*}$ are in-neighbors of $e$ in $D^{*}$. Thus, $\left|I_{2}^{*}\right| \leq \mu$ and hence $\left|I_{2}\right| \leq \mu$. Therefore,

$$
|I|=\left|I_{1}\right|+\left|I_{2}\right| \leq \mu+2 .
$$

So, the lemma follows.

Next, we describe a link ordering of $A$ under the 802.11 interference model with uniform interference radii, and two orientations of the conflict graphs under the 802.11 interference model and the protocol interference model respectively.

For the 802.11 interference model with uniform interference radii, we adopt the lexicographic ordering of $A$ defined below. We first define the lexicographic ordering of the nodes in $V$. For a pair of distinct nodes $u$ and $v$ in $V, u$ precedes $v$ in the lexicographic ordering if either $u$ lies strictly left to $v$ or they lie on the same vertical line but $u$ lies strictly below $v$. Second, we define the lexicographic ordering of the links in $A^{*}$. Consider a pair of distinct links $e$ and $e^{\prime}$ in $A^{*}$. Suppose that $u$ and $v$ are two endpoints of $e$ with $u$ preceding $v$ in the lexicographic ordering, and $u^{\prime}$ and $v^{\prime}$ are two endpoints of $e^{\prime}$ with $u^{\prime}$ preceding $v^{\prime}$ in the lexicographic order. If $e$ and $e^{\prime}$ have the same endpoints (i.e., $e^{\prime}$ is the reverse of $e$ ), then $e$ precedes $e^{\prime}$ in the lexicographic ordering if $e=(u, v)$ and $e^{\prime}=(v, u)$; otherwise, $e$ precedes $e^{\prime}$ in the lexicographic ordering if either $u$ preceding $u^{\prime}$ in the lexicographic ordering or $u^{\prime}=u$ but $v$ preceding $v^{\prime}$ in the lexicographic ordering. Third, we define the lexicographic ordering of the links in $A$. Consider a pair of distinct links $a$ and $a^{\prime}$ in $A$. Let $e$ and $e^{\prime}$ be their respective node-level links. If $e \neq e^{\prime}$ then $a$ precedes $a^{\prime}$ in the lexicographic ordering if $e$ precedes $e^{\prime}$ in the lexicographic ordering. If $e=e^{\prime}$, then $a$ precedes $a^{\prime}$ if either $a$ uses a smaller channel than $a^{\prime}$, or otherwise $a$ uses a smaller transmitting radio than $a^{\prime}$, or otherwise $a$ uses a smaller receiving radio than $a^{\prime}$.

Lemma 4: Under the 802.11 interference model with uniform interference radii, the lexicographic ordering of $A$ has BLIN at most 8.

Proof: The Proposition 5 in [5] implies that for each link $e$ in $A^{*}$, among all links in $A^{*}$ which conflict with $e$ and precede $e$ in the lexicographic ordering, at most 6 of them are independent. Hence, the BLIN of the lexicographic ordering of $A^{*}$ is at most 6 . Clearly, the lexicographic ordering of $A$ is compatible with the lexicographic ordering of $A^{*}$. By Lemma 2 , the BLIN of the lexicographic ordering of $A$ is at most $6+2=8$.

For the 802.11 interference model with arbitrary interference radii, we define an orientation $D$ of $G$ below. First, introduce an orientation $D^{*}$ of $G^{*}$ presented in [14]. For each node $v$, we use $\Gamma(v)$ to denote the set of nodes $w$ satisfying that

$$
\rho(w) \geq \max \{\rho(v),\|v w\|\}
$$


Consider a link $e=(u, v) \in A^{*}$ with $\rho(u) \geq \rho(v)$. It is said to be dominated by another link $e^{\prime} \in A^{*}$ if $e^{\prime}$ has an endpoint $w$ satisfying that either $w \in \Gamma(u)$, or $w \in \Gamma(v)$ and $\|u w\|>\max \{\rho(w), \rho(u)\}$. If $e$ is dominated by a link $e^{\prime}$, then $e^{\prime}$ is said to dominate $e$. It was proved in [14] that for any pair of conflicting links in $A^{*}$, at least one of them dominates the other. For any pair of conflicting links $e$ and $e^{\prime}$ in $A^{*}$, we orient the edge in $G^{*}$ between $e$ and $e^{\prime}$ to an arc in $D^{*}$ as follows: If they dominate each other, we take an arbitrary orientation; otherwise, if $e$ dominates $e^{\prime}$, we take the orientation from $e$ to $e^{\prime}$; otherwise, we take the orientation from $e^{\prime}$ to $e$. Second, we define the orientation $D$ of the conflict graph $G$. Consider any conflicting pair of links $a$ and $a^{\prime}$ in $A$. Let $e$ and $e^{\prime}$ be their respective node-level links. If $e \neq e^{\prime}$, then $e$ and $e^{\prime}$ conflict with each other. If $\left(e, e^{\prime}\right)$ is an arc in $D^{*}$, we take the orientation from $a$ to $a^{\prime}$ in $D$; otherwise, we take the orientation from $a^{\prime}$ to $a$ in $D$. If $e=e^{\prime}$, then we take the orientation from $a$ to $a^{\prime}$ in $D$ if either $a$ uses a smaller channel than $a^{\prime}$, or otherwise $a$ uses a smaller transmitting radio than $a^{\prime}$, or otherwise $a$ uses a smaller receiving radio than $a^{\prime}$.

Lemma 5: Under the 802.11 interference model, the orientation $D$ has ILIN at most 10 .

Proof: It was proved in [14] that the ILIN of the orientation $D^{*}$ of $G^{*}$ is at most 8. Clearly, the orientation $D$ of $G$ is compatible with the orientation $D^{*}$ of $G^{*}$. By Lemma 3, the ILIN of the orientation $D$ of $G$ is at most $8+2=10$.

For the protocol interference model, we define an orientation $D$ of $G$ below. First, introduce an orientation $D^{*}$ of $G^{*}$ presented in [13]. For tie-breaking, we assume that all nodes have distinct IDs. For any pair of conflicting links $e=(u, v)$ and $e^{\prime}=\left(u^{\prime}, v^{\prime}\right)$, if $v$ is within the interference range of $u^{\prime}$ and $v^{\prime}$ is within the interference range of $u$, take the orientation from $e$ to $e^{\prime}$ if and only if either $u$ has smaller ID than $u^{\prime}$ or $u=u^{\prime}$ but $v$ has smaller ID than $v^{\prime}$. If $v$ is within the interference range of $u^{\prime}$ but $v^{\prime}$ is outside the interference range of $u$, take the orientation from $e^{\prime}$ to $e$. If $v^{\prime}$ is within the interference range of $u$ but $v$ is outside the interference range of $u^{\prime}$, we take the orientation from $e$ to $e^{\prime}$. Second, we define the orientation $D$ of the conflict graph $G$. Consider any conflicting pair of links $a$ and $a^{\prime}$ in $A$. Let $e$ and $e^{\prime}$ be their respective node-level links. If $e \neq e^{\prime}$, then $e$ and $e^{\prime}$ conflict with each other and we take the orientation from $a$ to $a^{\prime}$ in $D$ if and only if $\left(e, e^{\prime}\right)$ is an arc in $D^{*}$. If $e=e^{\prime}$, then we take the orientation from $a$ to $a^{\prime}$ in $D$ if and only if either $a$ uses a smaller channel than $a^{\prime}$, or otherwise $a$ uses a smaller transmitting radio than $a^{\prime}$, or otherwise $a$ uses a smaller receiving radio than $a^{\prime}$.

Lemma 6: Under the protocol interference model, if the interference radius of each node is at least $c$ times its communication radius for some $c>1$, the orientation $D$ has ILIN at most $\left\lceil\pi / \arcsin \frac{c-1}{2 c}\right\rceil+1$.
Proof: It was proved in [13] that the ILIN of the orientation $D^{*}$ of $G^{*}$ is at most $\left\lceil\pi / \arcsin \frac{c-1}{2 c}\right\rceil+1$. Clearly, the orientation $D$ of $G$ is compatible with the orientation $D^{*}$ of $G^{*}$. By Lemma 3, the ILIN of the orientation $D$ of $G$ is at most

$$
\begin{aligned}
& \left\lceil\pi / \arcsin \frac{c-1}{2 c}\right\rceil-1+2 \\
& =\left\lceil\pi / \arcsin \frac{c-1}{2 c}\right\rceil+1 .
\end{aligned}
$$

So, the lemma holds.

\section{ApProximation Algorithms}

Let $(V, A, \mathcal{I})$ be the fine-grained network representation of a MC-MR multihop wireless network. A (fractional) link schedule is a set

$$
S=\left\{\left(I_{j}, \ell_{j}\right) \in \mathcal{I} \times \mathbb{R}_{+}: 1 \leq j \leq k\right\} .
$$

The value $\sum_{j=1}^{k} \ell_{j}$ is referred to as the length (or latency) of $S,|S|$ is called the size of $S$, and the function $c_{S} \in \mathbb{R}_{+}^{A}$ given by

$$
c_{S}(a)=\sum_{j=1}^{k} \ell_{j}\left|I_{j} \cap\{a\}\right|, \forall a \in A
$$

is called the link load function supported by $S$. If the length of $S$ is at most one, the function $c_{S}$ gives rise to a link capacity function. Suppose that we are given with a set of commodities in $(V, A)$. For any link schedule $S$ of length at most one, the maximum multiflow of these commodities subject to the capacity function $c_{S}$ is referred to as the maximum multiflow subject to $S$. Suppose in addition that each commodity also has a demand associated with it. For any link schedule $S$ of length at most one, the maximum concurrent multiflow of these commodities subject to the capacity function $c_{S}$ is referred to as the maximum concurrent multiflow subject to $S$. Note that for any fixed $S$, both maximum multiflow subject to $S$ and maximum concurrent multiflow subject to $S$ can be computed in polynomial time. The problem Maximum Multiflow (MMF) seeks a link schedule of length at most one which maximizes the total multiflow of a given a set of commodities. The problem Maximum Concurrent Multiflow (MCMF) seeks a link schedule of length at most one which maximizes the concurrent multiflow of a given a set of commodities together with demands. In this section, we present polynomial approximation algorithms for MMF and MCMF under the 802.11 interference model or the protocol interference model.

\section{A. Restricted Multiflow}

In this subsection, we present practical approximation algorithms for and following the polyhedral approach as described in [13]. For each node $v \in V$, we use $\delta^{i n}(v)$ (respectively, 
$\left.\delta^{\text {out }}(v)\right)$ to denote the set of links in $A$ entering (respectively, leaving) $v$. Consider two distinct nodes $s, t \in V$. A vector $f \in \mathbb{R}_{+}^{A}$ is called a flow from $s$ to $t$, or simply a $s-t$ flow, if for each $v \in V \backslash\{s, t\}$,

$$
f\left(\delta^{\text {out }}(v)\right)=f\left(\delta^{\text {in }}(v)\right) .
$$

This condition is called the flow conservation law: the amount of flow entering a vertex $v \neq s, t$ should be equal to the amount of flow leaving $v$. The value of a flow $f$ from $s$ to $t$ is, by definition:

$$
\operatorname{val}(f)=f\left(\delta^{\text {out }}(s)\right)-f\left(\delta^{\text {in }}(s)\right) .
$$

So, the value is the net amount of flow leaving $s$, which is also equal to the net amount of flow entering $t$. Suppose that we are given $k$ commodities with $s_{i}, t_{i}$ being the source and sink, respectively, for commodity $i$. We use $\mathcal{F}_{i}$ to denote the set of $s_{i}-t_{i}$ flows. A $k$-flow is a sequence of flows $\left\langle f_{1}, f_{2}, \cdots, f_{k}\right\rangle$ with $f_{i} \in \mathcal{F}_{i}$ for each $1 \leq i \leq k$.

Let $P$ be the convex hull of the incidence vectors of all independent sets in $\mathcal{I}$. Then, $P$ consists of all link capacity functions $c_{S}$ where $S$ is a fractional link schedule of length at most one. Thus, $P$ is also called the capacity region of the underlying wireless network. The maximum multiflow can be formulated as the following linear program (LP)

$$
\begin{array}{ll}
\max & \sum_{i=1}^{k} \operatorname{val}\left(f_{i}\right) \\
\text { s.t. } & f_{i} \in \mathcal{F}_{i}, \forall 1 \leq i \leq k ; \\
& \sum_{i=1}^{k} f_{i} \in P .
\end{array}
$$

Similarly, the maximum concurrent multiflow with demands $d(i)$ for $1 \leq i \leq k$ can be formulated as the following LP

$$
\begin{array}{ll}
\max & \phi \\
\text { s.t. } & f_{i} \in \mathcal{F}_{i}, \forall 1 \leq i \leq k ; \\
& \text { val }\left(f_{i}\right) \geq \phi d(i), \forall 1 \leq i \leq k ; \\
& \sum_{i=1}^{k} f_{i} \in P .
\end{array}
$$

In general, the membership of the capacity region $P$ is NPcomplete. In the restriction approach, we replace the capacity region $P$ by a subregion $Q$ of $P$ which satisfies the following three conditions: (1) $Q$ has an explicit representation by a polynomial number of linear inequalities or equalities, (2) there is a polynomial algorithm which produces a fractional schedule of length at most one for any $d \in Q$, and (3) $Q$ is a $\mu$-approximation of $P$ for some $\mu \geq 1$, i.e., $Q \subseteq P \subseteq$ $\mu Q$. The first condition ensures the membership of $Q$ is polynomial, the second condition implies that $Q \subseteq P$, and the third condition ensures that $Q$ is "close" to $P$. A capacity subregion $Q$ satisfying these three conditions is referred to as a polynomial $\mu$-approximate capacity subregion. The polynomial approximate capacity subregion plays a central role in the design and analysis of polynomial-time approximation algorithms. Suppose that $Q$ is a polynomial $\mu$-approximate capacity subregion. A $k$-flow $\left\langle f_{1}, f_{2}, \cdots, f_{k}\right\rangle$ is said to be
$Q$-restricted if $\sum_{i=1}^{k} f_{i} \in Q$. The maximum $Q$-restricted multiflow is defined by the following LP

$$
\begin{array}{ll}
\max & \sum_{i=1}^{k} \operatorname{val}\left(f_{i}\right) \\
\text { s.t. } & f_{i} \in \mathcal{F}_{i}, \forall 1 \leq i \leq k ; \\
& \sum_{i=1}^{k} f_{i} \in Q .
\end{array}
$$

This LP is of polynomial size and we solve this $Q$-restricted LP in polynomial time to obtain a $k$-flow $\left\langle f_{1}, f_{2}, \cdots, f_{k}\right\rangle$. Then we compute a fractional link schedule of length at most one for $\sum_{i=1}^{k} f_{i}$. Such link schedule is a $\mu$-approximate solution. Similarly, the maximum concurrent $Q$-restricted multiflow with demands $d(i)$ for $1 \leq i \leq k$ is defined by the following LP

$$
\begin{array}{ll}
\max & \phi \\
\text { s.t. } & f_{i} \in \mathcal{F}_{i}, \forall 1 \leq i \leq k ; \\
& \text { val }\left(f_{i}\right) \geq \phi d(i), \forall 1 \leq i \leq k ; \\
& \sum_{i=1}^{k} f_{i} \in Q .
\end{array}
$$

We first solve this $Q$-restricted LP of polynomial size in polynomial time to obtain a $k$-flow $\left\langle f_{1}, f_{2}, \cdots, f_{k}\right\rangle$, and then compute a fractional link schedule of length at most one for $\sum_{i=1}^{k} f_{i}$. This link schedule is a a $\mu$-approximate solution.

Therefore, if we can find a polynomial $\mu$-approximate capacity subregion, then both MMF and MCMF admit a polynomial $\mu$-approximation. Let $G$ be the conflict graph of $A$. Then, the independence polytope of $G$ is exactly the capacity region $P$, and the backward independence polytopes and inward independence polytopes of $G$ described in Section II are polynomial approximate capacity subregions. Specifically, we make use of the following three polynomial approximate capacity subregions.

- Under the 802.11 interference model with uniform interference radii, the lexicographic ordering of $A$ defined in Section III has BLIN at most 8 by Lemma 4. Hence, its backward independence polytope is a polynomial 8approximate capacity subregion by Theorem 1 .

- Under the interference model with arbitrary interference radii, the orientation $D$ of $G$ defined in Section III has ILIN at most 10 by Lemma 5 . Hence, its inward independence polytope is a polynomial 20-approximate capacity subregion by Theorem 1 .

- Under the protocol model with arbitrary interference radii, if the interference radius of each node is at least $c$ times its communication radius for some $c>$ 1 , the orientation $D$ of $G$ defined in Section III has ILIN at most $\left\lceil\pi / \arcsin \frac{c-1}{2 c}\right\rceil+1$ by Lemma 6 . Hence, its inward independence polytope is a polynomial $2\left(\left\lceil\pi / \arcsin \frac{c-1}{2 c}\right\rceil+1\right)$-approximate capacity subregion by Theorem 1 .

Following the restriction approach together with the above three polynomial approximate capacity subregions, we obtain the following two theorems. 
Theorem 7: Under the 802.11 interference model, both MMF and MCMF have a polynomial 20-approximation algorithm in general and a polynomial 8-approximation algorithm with uniform interference radii.

Theorem 8: Under the protocol interference model, if the interference radius of each node is at least $c$ times its communication radius for some $c>1$, both MMF and MCMF have a polynomial $2\left(\left\lceil\pi / \arcsin \frac{c-1}{2 c}\right\rceil+1\right)$-approximation algorithm.

\section{B. Polynomial-Time Approximation Schemes}

In this subsection, we establish the following approximation hardness of MMF and MCMF, which is the generalization to the SC-SR variant proved in [13].

Theorem 9: Suppose that the number of channels $\lambda$ is bounded by a constant, then both MMF and MCMF admit a PTAS when

1) under either the 802.11 interference model, or

2) under the protocol interference model in which the interference radius of each node is at least $c$ times its communication radius for some constant $c>1$, or

3) under either the 802.11 interference model in which every $h$-hop neighborhood in the conflict-graph contains at most $O\left(h^{c}\right)$ conflict-free links for some constant $c>0$.

We sketch the proof outline below. Given any link demand function $d \in \mathbb{R}_{+}^{A}$, the problem Maximum Weighted Independent Set of Links (MWISL) seeks a set $I \in \mathcal{I}$ with maximum $d(I)$. By applying ellipsoid method with (approximate) separation oracle as in [13], we can obtain the approximation-preserving reduction from MMF and MCMF to MWISL:

Theorem 10: If MWISL has a polynomial $\mu$-approximation algorithm, so do MMF and MCMF.

On the other hand, by utilizing the shifting strategy combined with dynamic programming as in [13], we can obtain a PTAS for MWISL.

Theorem 11: Suppose that the number of channels $\lambda$ is bounded by a constant, then MWISL admits a PTAS when

1) under either the 802.11 interference model, or

2) under the protocol interference model in which the interference radius of each node is at least $c$ times its communication radius for some constant $c>1$, or

3) under either the 802.11 interference model in which every $h$-hop neighborhood in the conflict-graph contains at most $O\left(h^{c}\right)$ conflict-free links for some constant $c>0$.

Theorem 9 then follows from Theorem 10 and Theorem 11 immediately.

\section{RELATED WORKS}

Maximum (concurrent) multiflow in SC-SR multihop wireless networks under 802.11/protocol interference model is now fairly well understood. Even when all nodes have uniform (and fixed) communication radii and uniform (and fixed) interference radii, it is NP-hard to compute a maximum (concurrent) multiflow [13]. On the other hand, polynomial approximation schemes (PTAS's) exist for maximum (concurrent) multiflow in a broad class of networks [13]. In addition to these hardness and approximation hardness results, a series of works have been devoted to practical approximation algorithms [1], [2], [4], [6], [8], [13], [15], [16]. Under either the 802.11 interference model with arbitrary interference and communication radii, the best known approximation bound is 23 [13]. Under the protocol interference model with arbitrary interference and communication radii, the best known approximation bound is $2\left(\left\lceil\pi / \arcsin \frac{c-1}{2 c}\right\rceil-1\right)$ where $c$ is the maximum ratio of the interference radius to communication radius of all nodes. Under the the 802.11/protocol interference model in which the communication radii of all nodes are equal to one and the interference radii of all nodes are equal to $\rho$ for some $\rho \geq 1$, a spatial divide-and-conquer approximation algorithm was developed in [13] with better approximation bounds. The approximation bound decreases with $\rho$ in general. For example, the approximation bound is at most 3 when $\rho \geq 2.2907$ under the 802.11 interference model or when $\rho \geq 4.2462$ under the protocol interference model.

In contrast, maximum (concurrent) multiflow in MC-MR multihop wireless networks have been studied by only a few research works [1], [3], [7], [10], [9], [11], [12]. Among these works, Alicherry et al. [1] proved an approximation bound $8 \lambda / \min _{v \in V} \tau(v)$ on their approximation algorithms under the 802.11 interference model with uniform interference radii. Such approximation bound grows linearly with the number of channels. Despite of lengthy effort in [1], it's fairly easy to achieve the same or even better approximation bound with a simple algorithm using only $\min _{v \in V} \tau(v)$ channels and $\min _{v \in V} \tau(v)$ radios per node. Such an algorithm is presented in the appendix of this paper. Lin and Rasool [10] studied the queuing-theoretic aspects of link scheduling in MC-MR multihop wireless networks under the 802.11 interference model with uniform interference/communication radii. Han et al. [10] gave an approximation algorithm for the maximum (concurrent) multiflow in MC-MR multihop wireless networks, which achieves an approximation bound of 122 under the 802.11 interference model with arbitrary interference/communication radii. We remark that the link scheduling algorithms used in both [1] and [10] suffer from the super-exponential worstcase running time as explained in [13]. In contrast to the quintuple representation of communication links in this paper, each communication link is represented by a triple of the transmitting node, the receiving node, and the channel in [10], or a duple of the transmitting radio and the receiving radio in [3]. Such subtle difference in the representations of the 
communication links turns to be very critical. Our fine-grained network representation allows for the definition of the conflict graph and the unified graph-theoretical treatment in the same way as in the SC-SR multihop networks, while the other two do not. We are then able to leverage the general techniques developed in [13] to obtain stronger and/or newer results in a simpler and cleaner manner. For practical wireless networks in which the number of channels is bounded by a constant, this paper also discovered the existence of PTAS for a number of link scheduling problems.

\section{CONCLUSION}

In this paper, we have conducted comprehensive studies on both MMF and MCMF in muliti-channel multi-radio multihop wireless networks under the 802.11 interference model. A fine-grained network representation and its associated conflict graph were proposed and their topological properties were explored. These structures and their properties are of independent interest and are expected to find applications in the studies of other problems in muliti-channel multi-radio multihop wireless networks. By exploiting these properties, we developed practical polynomial approximation algorithms for MMF and MCMF. Under the 802.11 interference model, their approximation bounds at most 8 with uniform interference radii and at most 20 in general and; under the protocol interference model, their approximation bounds are at most $2\left(\left\lceil\pi / \arcsin \frac{c-1}{2 c}\right\rceil-1\right)$ where $c=\min _{v \in V} \rho(v) / c(v)$. The approximation bound 8 is a significant improvement on the approximation bound $8 \lambda / \min _{v \in V} \tau(v)$ obtained by Alicherry et al. [1], which grows linearly with the number of channels. The latter two fill the blank of constant approximation bounds for MMF and MCMF in muliti-channel multi-radio multihop wireless networks. In addition, we also show that if the number of channels $\lambda$ is bounded by a constant, both MMF and MCMF admit a PTAS under the 802.11 interference model or under the protocol interference model with some additional mild conditions.

Two generalizations can be made to our studies. First, we have assumed that all radios can switch to any of the available channels. For the general case in which each radio can tune to only a subset of channels, we can handle it by putting the corresponding restriction on the radio-level links in the refined network representation. Specifically, for each link $e=(u, v) \in$ $A^{*}$, we only include those replications $\left(u, v, \gamma_{1}, \gamma_{2}, \gamma_{3}\right)$ for $1 \leq \gamma_{1} \leq \tau(u), 1 \leq \gamma_{2} \leq \tau(v)$, and $1 \leq \gamma_{2} \leq \lambda$ satisfying that both the $\gamma_{1}$-th radio at $u$ and the $\gamma_{2}$-th radio at $v$ can switch to the $\gamma_{3}$-th channel. With such modification, all properties and results stated in Section III and Section IV still holds.

Second, we have assumed all the channels have the same bandwidth normalized to one. Our approximation algorithms can be extended to the general settings with arbitrary link bandwidth function. Suppose that the link bandwidth function is given by positive vector $b \in \mathbb{R}_{+}^{A}$. For any $d \in \mathbb{R}_{+}^{A}$, we use $d \oslash b$ to denote the function in $\mathbb{R}^{A}$ defined by $(d \oslash b)(a)=$ $d(a) / b(a)$ for each $a \in A$. Then, our practical polynomial approximation algorithms described in Subsection IV-A can be modified by simply replacing the constraint

$$
\sum_{i=1}^{k} f_{i} \in Q
$$

with

$$
\left(\sum_{i=1}^{k} f_{i}\right) \oslash / b \in Q
$$

As in the single-channel single-radio multihop wireless networks [13], Theorem 10 on polynomial approximationpreserving reductions from MMF and MCMF to MWISL is also true in multi-channel multi-radio multihop wireless networks with arbitrary link bandwidth function. Hence, Theorem 9 on PTAS still holds in multi-channel multi-radio multihop wireless networks with arbitrary link bandwidth function.

Acknowledgements: The work of P.-J. Wan and Z. Wang described in this paper was supported in part by the NSF grants CNS-0831831 and CNS-0916666. The work of Y. Cheng described in this paper was supported in part by the NSF grants CNS-0832093 and CNS-1053777. The work of F. Yao described in this paper was partially supported by a grant from the Research Grants Council of the Hong Kong SAR, China under Project No.122807, and the National Basic Research Program of China Grant 2007CB807900, 2007CB807901 and 2011CBA00300, 2011CBA00302. The authors would like to thank the anonymous reviewers for their valuable suggestions and bringing to our attention the two technically relevant references [10] and [3] in the different context of queuingtheoretical studies and software-defined radio networks.

\section{REFERENCES}

[1] M. Alicherry, R. Bhatia, and L.E. Li, Joint Channel Assignment and Routing for Throughput Optimization in Multiradio Wireless Mesh Networks, IEEE Journal on Selected Areas in Communications 24(11):1960-1971, Nov. 2006. Also appeared in Proc. of ACM MobiCom, 2005.

[2] C. Buragohain, S. Suri, C. D. Toth, and Y. Zhou, Improved Throughput Bounds for Interference-Aware Routing in Wireless Networks, Proc. COCOON 2007, Lecture Notes in Computer Science 4598, 2007, pp. 210-221.

[3] B. Han, V. S. A. Kumar, M. V. Marathe, S. Parthasarathy, and A. Srinivasan, Distributed Strategies for Channel Allocation and Scheduling in Software-Defined Radio Networks, Proc. IEEE INFOCOM 2009, pp. 1521-1529.

[4] K. Jain, J. Padhye, V.N. Padmanabhan, and L. Qiu, Impact of interference on multi-hop wireless network performance, Proc. of ACM MobiCom, 2003.

[5] C. Joo, X. Lin, and N. B. Shroff, Understanding the capacity region of the greedy maximal scheduling algorithm in multi-hop wireless networks, in Proc. IEEE INFOCOM 2008, April 2008.

[6] M. Kodialam and T. Nandagopal, Characterizing achievable rates in multi-hop wireless networks: the joint routing and scheduling problem, Proc. of ACM MobiCom, 2003.

[7] M. Kodialam and T. Nandagopal. Characterizing the capacity region in multi-radio multi-channel wireless mesh networks, Proc. of ACM MobiCom, 2005. 
[8] V.S.A. Kumar, M.V. Marathe, S. Parthasarathy, and A. Srinivasan, Algorithmic aspects of capacity in wireless networks, SIGMETRICS Perform. Eval. Rev., 33(1):133-144, 2005.

[9] P. Kyasanur and N. Vaidya. Routing and Interface Assignment in MultiChannel Multi-Interface Wireless Networks, IEEE Wireless Communications and Networking Conference, New Orleans, LA, March 2005.

[10] X. Lin and S. Rasool, A Distributed Joint Channel-Assignment, Scheduling and Routing Algorithm for Multi-Channel Ad-hoc Wireless Networks, Proc. IEEE INFOCOM 2009, pp. 1118-1126

[11] A. Raniwala and T.-C. Chiueh, Architecture and algorithms for an IEEE 802.11-based multi-channel wireless mesh network, in Proc. IEEE INFOCOM, 2005, pp. 2223-2234.

[12] A. Raniwala, K. Gopalan, and T.-C. Chiueh, Centralized channel assignment and routing algorithms for multi-channel wireless mesh networks, ACM Mobile Comput. Commun. Rev. (MC2R), vol. 8, no. 2, pp. 50-65, 2004.

[13] P.-J. Wan, Multiflows in Multihop Wireless Networks, ACM MOBIHOC 2009, pp. 85-94.

[14] P.-J. Wan,C. Ma,Z. Wang, B. Xu, M. Li, and X. Jia, Weighted Wireless Link Scheduling without Information of Positions And Interference/Communication Radii, in Proc. IEEE INFOCOM, 2011.

[15] P.-J. Wan, L. Wang,A. Huang, M. Li,and F. Yao, Approximate Capacity Subregions of Uniform Multihop Wireless Networks, IEEE INFOCOM 2010.

[16] Y. Wang, W. Wang, X.-Y. Li, and W.-Z. Song, Interference-Aware Joint Routing and TDMA Link Scheduling for Static Wireless Networks, IEEE Transactions on Parallel and Distributed Systems 19(12): 17091726, Dec. 2008. An early version of this paper appeared in Proc. of ACM MobiCom, 2006.

\section{APPEndiX: NAIVE AlgorithmS}

In this section, we show that any polynomial $\mu$ approximation algorithm $\mathcal{A}^{*}$ for MMF (respectively, MCMF) in single-channel single-radio multihop wireless networks leads to a naive polynomial $\lambda \mu / \min _{v \in V} \tau(v)$-approximation algorithm for MMF (respectively, MCMF) in multi-channel multi-radio multihop wireless networks. We only describe the algorithm for MMF, as the algorithm for MCMF is similar.

We first apply the algorithm to the single-channel singleradio network $\left(V, A^{*}, \mathcal{I}^{*}\right)$ to obtain a fractional link schedule

$$
S^{*}=\left\{\left(I_{j}^{*}, \ell_{j}^{*}\right) \in \mathcal{I}^{*} \times \mathbb{R}_{+}: 1 \leq j \leq q\right\},
$$

and obtain a $k$-flow $\left\langle f_{1}^{*}, f_{2}^{*}, \cdots, f_{k}^{*}\right\rangle$ for the given $k$ commodities. For each $e=(u, v) \in A^{*}$, denote by $\Gamma^{\prime}(e)$ the set of $\min _{v \in V} \tau(v)$ links in $A$ from $u$ to $v$, the $i$-th of which uses the $i$-th radio at $u$, the $i$-th radio at $v$ and the $i$-th channel for each $1 \leq i \leq \min _{v \in V} \tau(v)$. The set $\Gamma^{\prime}(e)$ can be regarded as a $\min _{v \in V} \tau(v)$-fold replication of $e$. For each $1 \leq j \leq q$, let

$$
I_{j}=\cup_{e \in I_{j}^{*}} \Gamma^{\prime}(e) .
$$

Then, the set

$$
S=\left\{\left(I_{j}, \ell_{j}^{*}\right): 1 \leq j \leq q\right\}
$$

is a fractional link schedule in $(V, A, \mathcal{I})$ with the same length as $S^{*}$. In addition, it can support the $k$-flow $\left\langle f_{1}, f_{2}, \cdots, f_{k}\right\rangle$ given by

$$
f_{i}(a)=f_{i}^{*}(e), \forall a \in \Gamma^{\prime}(e), e \in A^{*}, 1 \leq i \leq k .
$$

The $k$-flow $\left\langle f_{1}, f_{2}, \cdots, f_{k}\right\rangle$ can also be regarded as a $\min _{v \in V} \tau(v)$-fold replication of the $k$-flow $\left\langle f_{1}^{*}, f_{2}^{*}, \cdots, f_{k}^{*}\right\rangle$. Clearly,

$$
\sum_{i=1}^{k} \operatorname{val}\left(f_{i}\right)=\min _{v \in V} \tau(v) \sum_{i=1}^{k} \operatorname{val}\left(f_{i}^{*}\right) .
$$

Next, we show that the $k$-flow $\left\langle f_{1}, f_{2}, \cdots, f_{k}\right\rangle$ is a $\lambda \mu / \min _{v \in V} \tau(v)$-approximate solution.

Consider an optimal link schedule

$$
O P T=\left\{\left(I_{j}^{\prime}, \ell_{j}^{\prime}\right): 1 \leq j \leq q^{\prime}\right\}
$$

and the associated maximum $k$-flow $\left\langle g_{1}, g_{2}, \cdots, g_{k}\right\rangle$. For each $1 \leq j \leq k$ and each $1 \leq l \leq \lambda$, let $I_{j l}$ denote the set of nodelevel links of the links in $I_{j}$ using the channel $l$. Then, the set

$$
O P T^{*}=\left\{\left(I_{j l}, \ell_{j} / \lambda\right): 1 \leq j \leq q^{\prime}, 1 \leq l \leq \lambda\right\}
$$

is a link schedule of length at most one in $\left(V, A^{*}, \mathcal{I}^{*}\right)$. In addition, it can support the $k$-flow $\left\langle g_{1}^{*}, g_{2}^{*}, \cdots, g_{k}^{*}\right\rangle$ given by

$$
g_{i}^{*}(e)=\frac{1}{\lambda} \sum_{a \in \Gamma(e)} g_{i}(e), \forall e \in A^{*}, \forall 1 \leq i \leq k .
$$

Clearly,

$$
\sum_{i=1}^{k} \operatorname{val}\left(g_{i}^{*}\right)=\frac{1}{\lambda} \sum_{i=1}^{k} \operatorname{val}\left(g_{i}\right) .
$$

Since $\mathcal{A}^{*}$ is a $\mu$-approximation algorithm, we have

$$
\sum_{i=1}^{k} \operatorname{val}\left(f_{i}^{*}\right) \geq \frac{1}{\mu} \sum_{i=1}^{k} \operatorname{val}\left(g_{i}^{*}\right) \geq \frac{1}{\lambda \mu} \sum_{i=1}^{k} \operatorname{val}\left(g_{i}\right) .
$$

Therefore,

$$
\begin{aligned}
\sum_{i=1}^{k} \operatorname{val}\left(f_{i}\right) & =\min _{v \in V} \tau(v) \sum_{i=1}^{k} \operatorname{val}\left(f_{i}^{*}\right) \\
& \geq \frac{\min _{v \in V} \tau(v)}{\lambda \mu} \sum_{i=1}^{k} \operatorname{val}\left(f_{i}^{*}\right) .
\end{aligned}
$$

So, the $k$-flow $\left\langle f_{1}, f_{2}, \cdots, f_{k}\right\rangle$ is a $\lambda \mu / \min _{v \in V} \tau(v)$ approximate solution.

We remark that the naive algorithm described above utilizes only $\min _{v \in V} \tau(v)$ channels and $\min _{v \in V} \tau(v)$ radios per node. Yet, by choosing the proper algorithm $\mathcal{A}^{*}$ such as the one given in [13], it can beat the approximation bound obtained by Alicherry et al. [1] under the 802.11 interference model with uniform interference radii. 